\title{
Design Considerations for the Manufacture of Temperature Stable Periodically-Poled Nonlinear Crystals
}

\author{
Huw E. Major, Anna C. Peacock, Corin B.E. Gawith ${ }^{1,2}$, Peter G.R. Smith ${ }^{1,2}$ \\ 1. Optoelectronics Research Centre (ORC), University of Southampton, Southampton, SO17 1BJ, United Kingdom \\ 2. Stratophase Ltd., Unit A7 Premier Centre, Romsey, SO51 9DG, United Kingdom
}

Second harmonic generation via periodically-poled nonlinear materials offers an efficient means of generating highquality visible light that would be otherwise unattainable with traditional laser sources. In particular the higher conversion efficiencies associated with periodically poled materials, such as lithium niobate, make them ideal for integration with lower power diode lasers. The combination of low-cost sources with high quality crystals is attracting interest for implementation in many mass-industrial applications, such as RGB TVs [1] and projectors. Setting aside the well known photorefractive issues, a remaining challenge is that the typical temperature stability requirements of $0.1^{\circ} \mathrm{C}$ can make packaging with a pump source problematic.

To reduce the stringent temperature control required for efficient operation of such devices we demonstrate a technique for producing constant SHG power over a broad temperature range, with a temperature acceptance bandwidth of up to $5^{\circ} \mathrm{C}$. The increased bandwidth is achieved by mapping the domain density of a constant period QPM grating to an apodised sinc function, the fourier transform of which results in a flat-top response (Fig.1a). By adjusting the domain density to match the intensity profile, including appropriate phase changes, it is possible to build a physical representation of the function. Incorporating physical limits such as constant domain size and minimum separation ensures a high fabrication yield.

Based on the resulting mathematical models and our high fidelity poling technique we have achieved precise placement of $3 \mathrm{um}$ poled domains in lithium niobate (Fig.2). These initial devices allow green SHG with up to $5^{\circ} \mathrm{C}$ flattop temperature stability, albeit with a corresponding loss in operational efficiency due to reduced domain density.

(a)

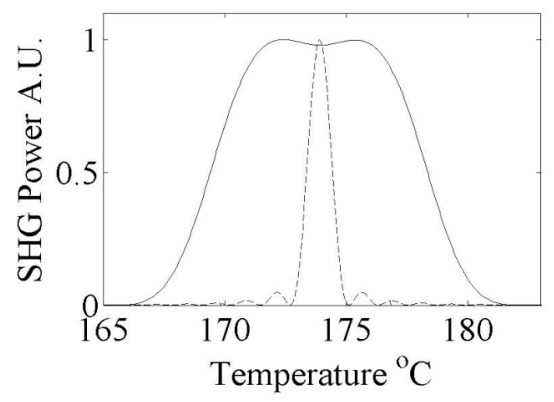

(b)

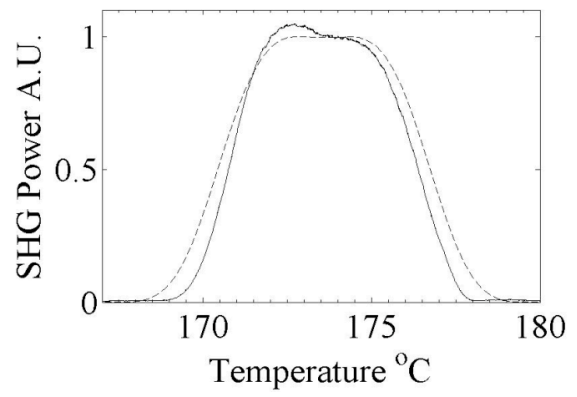

Fig 1. (a) Normalised SHG response of a $5^{\circ} \mathrm{C}$ flat top device (solid) and equivalent length PPLN (dashed) (b) Asymmetry profile of $3^{\circ} \mathrm{C}$ device (solid) against theory (dashed).

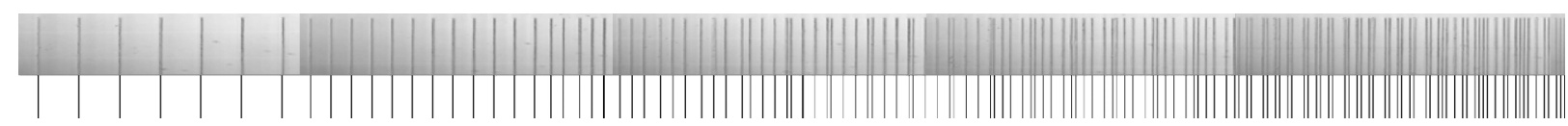

Fig 2. Section of poled grating with $3^{\circ} \mathrm{C}$ stability (top) with mask pattern shown for reference (bottom).

Upon testing it becomes apparent that focussing conditions can greatly affect device performance, with tight focussing leading to an asymmetric temperature tuning profile (Fig.1b). As the maximum efficiency of bulk devices demands a tighter focus, we are developing optimised grating designs using a modified version of the Boyd and Kleinman [2] formulation, which models focussing effects in nonlinear crystals. Taking this approach to intentionally introduce an opposing asymmetry and denser domain layout to our existing grating designs, we are developing higher-efficiency temperature-stable SHG devices that maintain flat-top operation under optimal focussing conditions. Ultimately, implementing these improved grating layouts in magnesium-doped lithium niobate to alleviate photorefraction will provide optimised solutions for packaging with near-room temperature diode-based sources.

\section{References}

1. www.novalux.com

2. G.D. Boyd \& D.A. Kleinman "Parametric Interaction of Focussed Gaussian Light Beams" J. Appl. Phys. 39, 3597-

$3639(1968)$ 